\title{
Exciton dynamics of a single quantum dot embedded in a nanowire
}

\author{
G. Sallen, ${ }^{1}$ A. Tribu, ${ }^{2}$ T. Aichele, ${ }^{1, *}$ R. André,${ }^{1}$ L. Besombes,${ }^{1}$ C. Bougerol,${ }^{1}$ S. Tatarenko, ${ }^{1}$ K. Kheng, ${ }^{2}$ and J. Ph. Poizat ${ }^{1, \dagger}$ \\ ${ }^{1}$ Institut Néel, CNRS-Université Joseph Fourier, 38042 Grenoble, France \\ ${ }^{2}$ CEA/INAC/SP2M, 38054 Grenoble, France
}

(Received 17 March 2009; revised manuscript received 27 July 2009; published 18 August 2009)

\begin{abstract}
We have carried out a detailed optical characterization of a single CdSe quantum dot embedded in a $\mathrm{ZnSe}$ nanowire. Exciton, biexciton, and charged exciton lines have been identified unambiguously using photon correlation spectroscopy. This technique has provided a detailed picture of the dynamics of this system. It has been found that the dark exciton has a strong influence on the optical properties. The most visible influence is the strongly reduced excitonic emission compared to the biexcitonic one. Temperature-dependent lifetime measurements have allowed us to measure a large splitting of $\Delta E=(6.0 \pm 0.2) \mathrm{meV}$ between the dark and the bright exciton as well as the spin-flip rates between these two states. This type of semiconducting quantum dot turns out to be a very efficient single photon source in the visible. Its particular growth technique opens additional possibilities as compared to the usual self-assembled quantum dots.
\end{abstract}

DOI: $10.1103 /$ PhysRevB.80.085310

PACS number(s): 78.67.Lt, 78.55.Et

\section{INTRODUCTION}

Semiconductor nanowires (NWs) appear as promising building blocks for nanoscale devices and circuits with impressive potential applications including nanoelectronics, ${ }^{1-3}$ optoelectronics (light-emitting $\operatorname{diodes}^{4,5}$ and nanolasers ${ }^{6}$ ), thermoelectrical energy conversion, ${ }^{7}$ and biological or chemical sensors. ${ }^{8}$ Moreover, high quality defect free NWs can be grown on low-cost routinely used substrates such as silicon, which means that they could easily be used for fabricating commercial devices and could possibly be integrated with mainstream Si microelectronics devices.

NW growth methods allow for the variation in the chemical composition ${ }^{9,10}$ or doping ${ }^{11}$ along the longitudinal or radial directions. This enables the fabrication of well controlled one-dimensional nanoscale heterostructures. ${ }^{10}$ For example, as shown in this work, it is possible to insert a slice of a low band-gap semiconductor within a high band-gap NW and thus realize a light-emitting quantum dot (QD) operating as a single photon source. ${ }^{12,13}$ So far, work on the light-emitting properties of single QDs has mainly concerned self-assembled QDs formed by surface forces induced by lattice mismatch between different materials. Such QDs have been widely used in the past decade as single photon sources ${ }^{14}$ and for their potential application in quantum information processing (see, for example, Refs. 15 and 16). QDs in NWs appear to be an interesting alternative to selfassembled QDs. The absence of a wetting layer offers a better confinement which could enable room-temperature production of single photons. ${ }^{13}$ Radial growth techniques enable engineering of optical guides allowing more efficient light extraction than in bulk materials. ${ }^{17,18}$ Furthermore, NWbased heterostructures, being much less limited by lattice mismatches, greatly widen the possible materials combinations and enable well controlled stacking of several QDs in a single NW, offering interesting possibilities for quantum information processing. ${ }^{19}$

In this paper we present a detailed optical characterization of excitonic emission in a single CdSe QD embedded in a $\mathrm{ZnSe}$ NW. We have already shown that this system is an efficient single photon source operating at temperatures as high as $220 \mathrm{~K} .{ }^{13}$ Single photon emission from NWs has otherwise only been demonstrated at $4 \mathrm{~K}$ in InAs QDs embedded in InP NWs. ${ }^{12}$ Our system emits light around $550 \mathrm{~nm}$ $(2.2 \mathrm{eV})$ where silicon avalanche photodiodes (APDs) are very efficient. This has allowed us to perform a thorough spectroscopic analysis of a QD embedded in a NW by using photon correlation spectroscopy. ${ }^{20}$ We have identified unambiguously the exciton, biexciton, and charged exciton lines and obtained information on the charging dynamics of this QD (Sec. III). We have fitted the various correlation functions with a model including the dark exciton and its parameters defined by lifetime measurements. The most apparent manifestation of the dark exciton is the large predominance of the biexciton line with respect to the exciton line above saturation. The dark exciton is studied in Sec. IV.

\section{SAMPLE AND EXPERIMENTAL SETUP}

\section{A. Sample fabrication and preparation}

The wires are grown by molecular beam epitaxy in the vapor-liquid-solid growth mode catalyzed by gold particles on a Si substrate. In order to make QDs, a small region of CdSe is inserted in the $\mathrm{ZnSe} \mathrm{NW}$. This is done by interrupting the $\mathrm{ZnSe}$ growth, changing to CdSe growth for a short time, and then growing ZnSe again. ${ }^{13}$ Details about the growth of the ZnSe NWs can be found in Ref. 21. The wire diameter (around $10 \mathrm{~nm}$ ) is on the order of the bulk exciton Bohr diameter for CdSe $(11 \mathrm{~nm})$. This means that the carriers in the CdSe QD are in the strong quantum confinement regime. For the study of single NWs, the sample is sonicated in methanol causing NWs to break off the substrate into the solution. Droplets of this solution are then deposited on a Si substrate and a low density of individual NWs is obtained after evaporation. As shown in Fig. 1, individual NWs can be isolated allowing single QD optical spectroscopy.

\section{B. Experimental setup}

The experimental apparatus is a standard microphotoluminescence ( $\mu \mathrm{PL})$ setup. The samples are mounted on a $X Y Z$ 


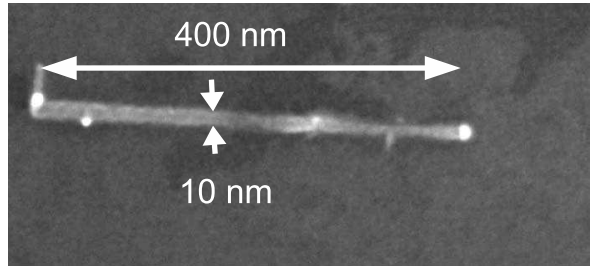

FIG. 1. Scanning electron microscope image of a single CdSe/ $\mathrm{ZnSe}$ nanowire deposited on a silicon substrate.

piezomotor system in a He flow cryostat at a temperature of $4 \mathrm{~K}$. The optical excitation is provided by a $405 \mathrm{~nm}$ continuous-wave diode laser illuminating the sample via a microscope objective of numerical aperture NA=0.65 located in the cryostat. Time-resolved measurements are performed by exciting the sample at a wavelength of $\lambda$ $=440 \mathrm{~nm}$ with a frequency doubled Ti:sapphire laser operating at $\lambda=880 \mathrm{~nm}$ with pulse duration of $1 \mathrm{ps}$ and a repetition rate of $80 \mathrm{MHz}$. The NW emission is collected by the same objective and sent to a 50/50 beam splitter for correlation measurements. In each arm of the beam splitter, the light is dispersed by a monochromator (1200 grooves/mm grating, $30 \mathrm{~cm}$ and $50 \mathrm{~cm}$ focal length, respectively). Each monochromator has a switchable mirror inside, which can direct the luminescence either onto a charge coupled device camera for the measurement of the PL spectrum or through the exit slit toward a low jitter (40 ps), high quantum efficiency APD. The detectors send electrical pulses into a time-correlated single photon module that builds a histogram of the time delays between successive photons. This histogram is proportional to the second-order correlation function $g^{(2)}(t) .{ }^{14}$ The overall temporal resolution of our setup is essentially limited by the jitter of the APDs and the dispersion of the monochromator gratings. This time resolution was measured by recording the autocorrelation function of 1 ps pulses from a frequency-doubled Ti:sapphire laser. A full width at half maximum of 90 ps was obtained for the autocorrelation function peak. For lifetime measurement, the timing resolution was measured by sending the 1 ps laser pulses in a monochromator and a full width at half maximum of 70 ps was obtained.

\section{General optical properties}

A typical low-temperature $\mu \mathrm{PL}$ spectrum is shown in the inset of Fig. 2 where three lines can be seen. A comparison with relative energy positions of known emission lines in spectra of self-assembled CdSe/ZnSe QDs (Refs. 22 and 23) suggests that these lines correspond to the exciton $(\mathrm{X})$, the biexciton $(\mathrm{XX})$, and the charged exciton $(\mathrm{CX})$. The $\mathrm{X}-\mathrm{CX}$ (resp. X-XX) energy splitting is found around $10 \mathrm{meV}$ (resp. $20 \mathrm{meV}$ ) as compared to 15-22 meV (resp. 19-26 $\mathrm{meV}$ ) for self-assembled QDs. The mean excitonic energy, $(2.25 \pm 0.08) \mathrm{eV}$, is also similar as compared to $(2.45 \pm 0.2) \mathrm{eV}$ for self-assembled CdSe/ZnSe QDs. ${ }^{24} \mathrm{Un}-$ ambiguous proof for the assignment of these lines will be given below using photon correlation spectroscopy. The linewidth broadening can be attributed to spectral diffusion. ${ }^{25}$ This effect might arise from the close proximity of the QD to

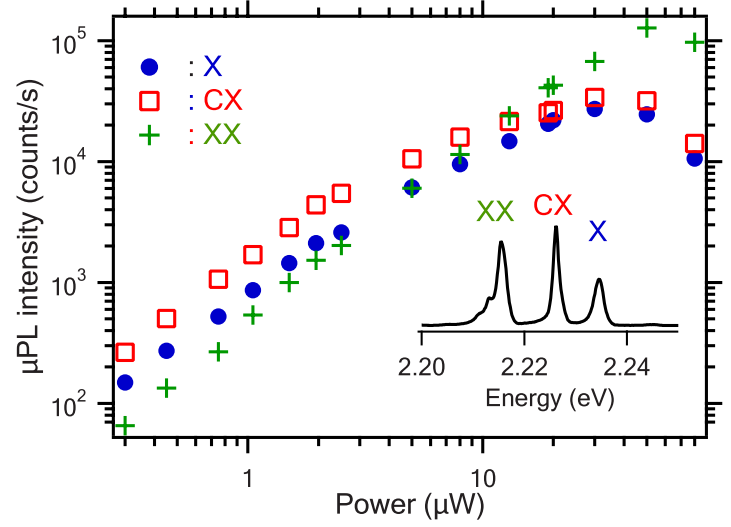

FIG. 2. (Color online) Inset: $\mu \mathrm{PL}$ spectrum of a nanowire quantum dot at an excitation power of $P=15 \mathrm{~W}$. The electronic background noise has been subtracted. Main plot: line intensities as a function of excitation power. The spectra were obtained at $4 \mathrm{~K}$.

the NW surface, where fluctuating charges can be trapped on surface states even at low temperature. ${ }^{12,26}$

One of the characteristic features of such NW QD structures is their polarization properties. As shown in Ref. 13 the excitation efficiency and the luminescence are both strongly polarization dependent. ${ }^{27-29}$ A $90 \%$ contrast is obtained for the excitation efficiency depending on the direction of the linear polarization of the pumping laser. The light emission is also $90 \%$ linearly polarized in the same direction as the best pumping polarization, independently of the excitation polarization.

It can be seen in Fig. 2 that the saturation level of the XX line is more than three times larger than that of the $\mathrm{X}$ line. As it will be shown in Sec. IV, this is due to a strong storage effect of the dark exciton state.

\section{PHOTON CORRELATION SPECTROSCOPY}

The results presented in this section all come from the same QD whose spectrum is shown in Fig. 2. The level scheme used to model our system is shown in Fig. 3. It is based on models used in Refs. 30-32 where the carriers can enter the QD either individually (power-dependent rates $\gamma_{C 1}$ and $\gamma_{C 2}$ ) or already bound as excitons (power-dependent rate

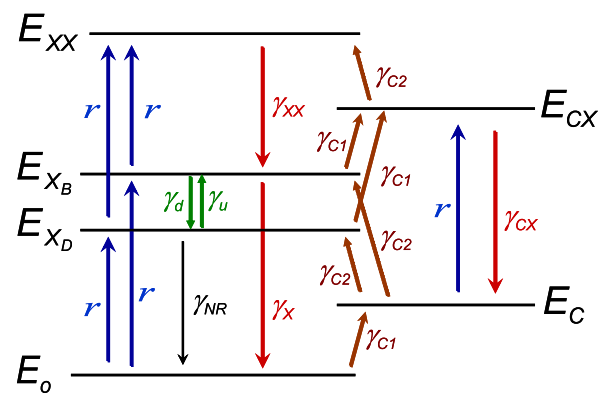

FIG. 3. (Color online) Level scheme including the empty dot $\left(E_{0}\right)$, the dark exciton $\left(E_{\mathrm{X}_{D}}\right)$, the bright exciton $\left(E_{\mathrm{X}_{B}}\right)$, the biexciton $\left(E_{\mathrm{XX}}\right)$, the charged $\operatorname{dot}\left(E_{C}\right)$, and the charged exciton $\left(E_{\mathrm{CX}}\right)$. The various rates between the different levels are indicated in the figure. 
TABLE I. Table of power-independent transition rates in $\mathrm{ns}^{-1}$ at a temperature $T=4 \mathrm{~K}$.

\begin{tabular}{cccccc}
\hline \hline$\gamma_{\mathrm{X}}$ & $\gamma_{\mathrm{XX}}$ & $\gamma_{\mathrm{CX}}$ & $\gamma_{d}$ & $\gamma_{u}$ & $\gamma_{\mathrm{NR}}$ \\
\hline 1.4 & 2.5 & 1.7 & 1.4 & 0 & 0.2 \\
\hline \hline
\end{tabular}

$r$ ). We have added the dark exciton, which plays a key role in our system. It includes the bright and dark exciton, the charged exciton, and the biexciton. A triexciton level and a charged biexciton level are also included to avoid artificial saturation of the biexciton and of the charged exciton but they are not represented in Fig. 3.

All of the power-independent parameters of the model can be evaluated independently prior to photon correlation experiments by performing lifetime measurements (see following Sec. IV). Their values are listed in Table I. These values are compatible to observations in self-assembled $\mathrm{CdSe} / \mathrm{ZnSe}$ QDs. $^{23}$

We come now to the results of this work on photon correlation experiments. We present first the data concerning the neutral QD in Fig. 4. The autocorrelation of the X line emission is shown in Fig. 4(a) exhibiting a clear antibunching which is characteristic of the statistics of a single photon emitter. ${ }^{14}$

Figure 4(b) shows the cross-correlation measurement between the $\mathrm{X}$ and the $\mathrm{XX}$ line. It displays the typical asymmetric shape with bunching and antibunching features that is the signature for the cascaded emission of a XX photon followed by a $\mathrm{X}$ photon. ${ }^{33}$ This allows us to identify unambiguously these two lines as exciton and biexciton of the same QD. Note that the narrow bunching peak can only be fitted if the dark exciton is included in the model.

For all the correlation graphs (Figs. 4 and 5) the right

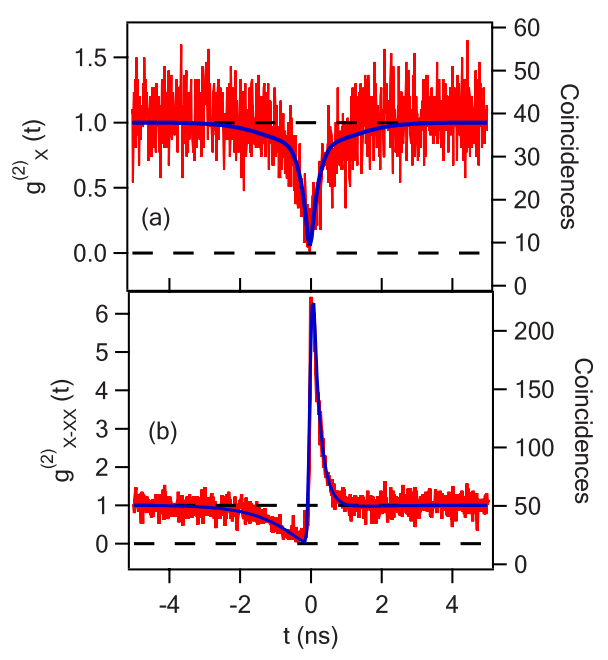

FIG. 4. (Color online) (a) Exciton emission autocorrelation, (b) exciton-biexciton cross correlation for an excitation power $P$ $=15 \mu \mathrm{W}$. The left axes are the correlation functions corrected from the background and the right axes are the raw coincidence rates (see text). The fit is performed using the model based on Fig. 3. The power-dependent parameters used for the fit are $r=0.6 \mathrm{~ns}^{-1}, \gamma_{C 1}$ $=\gamma_{C 2}=1 \mathrm{~ns}^{-1}$. The other parameters are given in Table I.

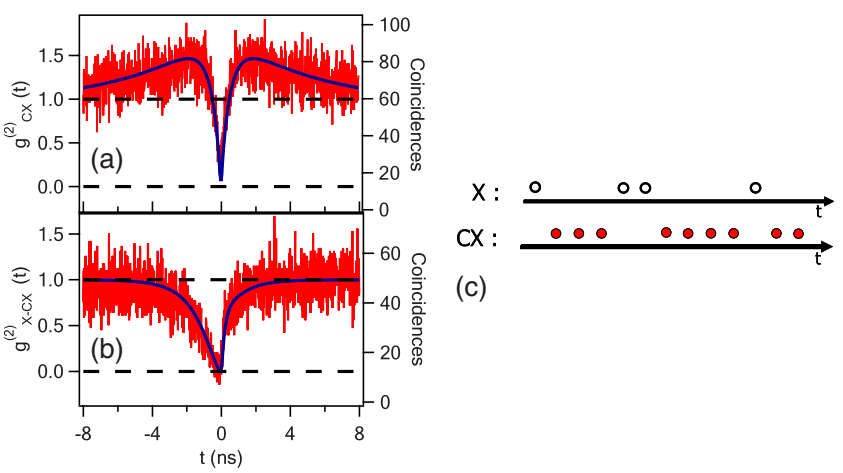

FIG. 5. (Color online) (a) Charged exciton autocorrelation for an excitation power $P=8 \mu \mathrm{W}$; (b) exciton-charged exciton cross correlation for an excitation power $P=10 \mu \mathrm{W}$. The fits are performed using the model based on Fig. 3. The power-dependent parameters used for the fits are, respectively, $r=0.31 \mathrm{~ns}^{-1}, \gamma_{C 1}=0.09 \mathrm{~ns}^{-1}$, and $\gamma_{C 2}=0.058 \mathrm{~ns}^{-1}$ for the CX autocorrelation (a) and $r=0.37 \mathrm{~ns}^{-1}$, $\gamma_{C 1}=0.25 \mathrm{~ns}^{-1}$, and $\gamma_{C 2}=0.28 \mathrm{~ns}^{-1}$ for the X-CX cross correlation (b). The other parameters are given in Table I. (c) Representation of the stream of photons coming alternatively from the neutral and the charged QD.

vertical axes are the raw number of coincidences. The left axes represent the normalized correlation function according to a Poisson statistics where the coincidences involving background photons have been subtracted. The corrected correlation function $g^{(2)}$ is related to the uncorrected one $g_{u}^{(2)}$ by $g^{(2)}-1=\left(g_{u}^{(2)}-1\right) / \rho^{2}$, where $\rho=S /(S+B)$ with $S$ and $B$, respectively, the number of signal and background photons as measured in the spectrum of Fig. 2.

The autocorrelation of the CX line is shown in Fig. 5(a). As for the $\mathrm{X}$ line, it exhibits a clear antibunching. Also, on a larger time scale, a bunching effect can be observed. This is due to the hopping from the charged state to the neutral state of the QD as confirmed by the X-CX cross correlation displayed in Fig. 5(b). This proves that the CX line comes from the same QD as the $\mathrm{X}$ line. The situation is depicted schematically in Fig. 5(c), which shows that the photons are emitted either from the charged or from the neutral state of the QD. The average time spent by the QD in the charged state is given by the characteristic time of an exponential fitting of the bunching peak in Fig. 5(a) which is 5 ns. It should be noticed that the antibunching dip in Fig. 5(b) is not symmetrical. Negative (positive) time corresponds to the probability of detecting a $\mathrm{CX}(\mathrm{X})$ photon after having detected a $\mathrm{X}(\mathrm{CX})$ photon. Formation of a $\mathrm{CX}$ in an empty QD $(t<0)$ requires the loading of three charges, whereas the formation of an $\mathrm{X}$ in a QD with a single charge (level $E_{c}$ in Fig. 3) is faster since it requires only the loading of a single charge $(t>0) .^{30-32}$ Although we do not know whether this charge is positive or negative, comparison with spectra of self-assembled CdSe/ZnSe QDs (Refs. 22 and 23) leads us to the assumption that the charge state is negative.

We also show in Fig. 6 the autocorrelation of the biexcitonic line and the cross correlation between the biexciton and the charged exciton. The biexciton autocorrelation exhibits a narrow antibunching and the $\mathrm{XX}-\mathrm{CX}$ cross-correlation features a broad antibunching caused by charge hopping as in the X-CX cross correlation of Fig. 5(b). 


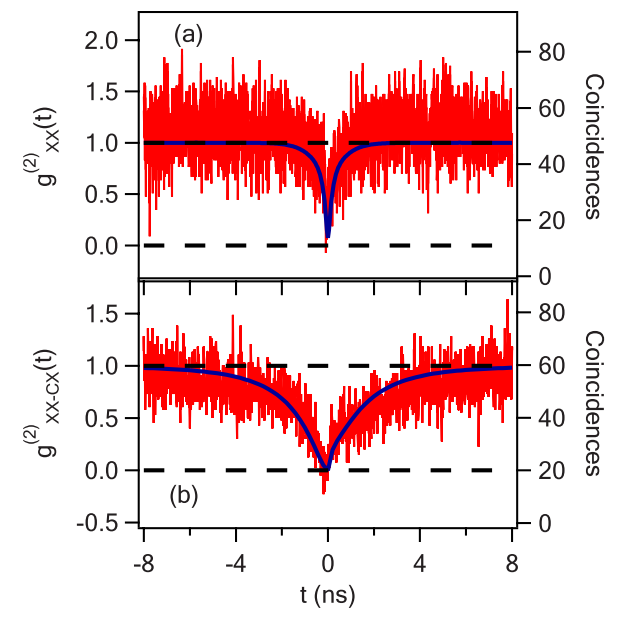

FIG. 6. (Color online) (a) Autocorrelation of the biexcitonic line (XX) for an excitation power of $P=15 \mu \mathrm{W}$, (b) biexciton-charged exciton cross correlation, both for an excitation power $P=15 \mu \mathrm{W}$. The fits are performed using the model based on Fig. 3. The powerdependent parameters used for the fits are $r=0.6 \mathrm{~ns}^{-1}, \quad \gamma_{C 1}$ $=1 \mathrm{~ns}^{-1}$, and $\gamma_{C 2}=2 \mathrm{~ns}^{-1}$. The other parameters are given in Table I.

As can be seen in Figs. 4-6, the experimental results are fitted very well by the model shown in Fig. 3 taking into account the temporal resolution of our experimental setup (90 ps). Inclusion of the dark exciton turned out to be essential for the modeling of the photon correlation data. Allowing for the coexistence of two excitation mechanisms, namely, charge by charge (described by $\gamma_{C 1}$ and $\gamma_{C 2}$ ) or directly feeding the QD with an already bound exciton (described by $r$ ) has also turned out to be necessary for the fitting. The QD charge hopping time depends on the value of these parameters. The model gives also the correct intensities of the spectral lines within $10 \% .^{25}$

\section{DARK EXCITON CHARACTERIZATION}

In a QD, lowest energy excitons are the combination of an electron $(\operatorname{spin} \pm 1 / 2)$ and a heavy hole $(\operatorname{spin} \pm 3 / 2)$. This results in two different energy levels of spin \pm 1 and spin \pm 2 . The spin- \pm 1 states are optically connected to the zero spin empty dot state and called the bright exciton. The low energy spin \pm 2 states are called the dark exciton because they are not optically active. Indeed a photon is a spin-1 particle that cannot carry away 2 quanta of angular momentum.

In this section we have performed temperature-dependent lifetime measurements on this $\mathrm{CdSe} / \mathrm{ZnSe} \mathrm{NW}$. By fitting these data with a model involving an acoustic phonon bath ${ }^{34}$ we are able to extract the value of the dark and bright exciton energy splitting $\Delta E$ and the spin-flip rates between these two states. The measurements performed in this section have been carried out on a QD with a similar spectrum but different than for the photon correlation results.

The ratio between charged and neutral QD luminescence is varying from dot to dot. We have also observed that increasing the temperature tends to neutralize the QD. The

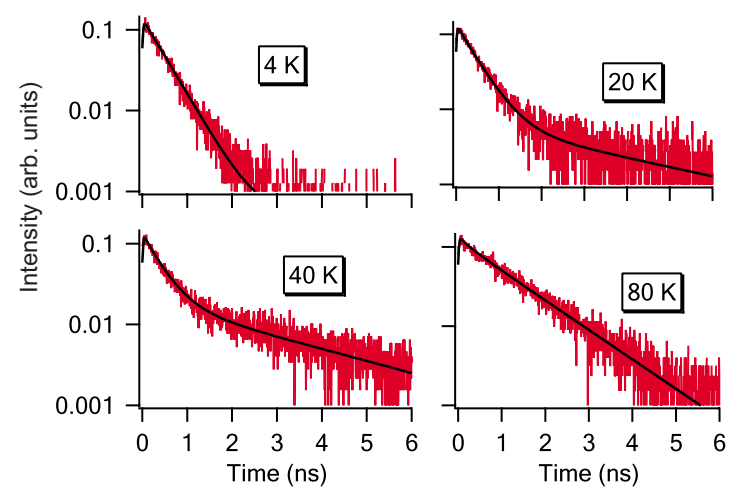

FIG. 7. (Color online) Decay of the X line emission of QD1 for different temperatures. The pumping power is well below saturation $\left(r \ll \gamma_{\mathrm{X}}\right)$ so that the XX state is almost not populated.

large linewidths have been attributed to spectral diffusion. ${ }^{25}$ The most conspicuous feature is that the $\mu \mathrm{PL}$ intensity of the $\mathrm{XX}$ line at saturation is always a lot larger than that of the $\mathrm{X}$ line as it is shown in the power dependence of the different lines (Fig. 2). This effect is the signature of a strong storage effect on the dark exciton (DX) state. The DX state reduces the luminescence of the $\mathrm{X}$ line owing to the leakage from the bright to the dark exciton but the DX state remains an efficient intermediate state for populating the XX state. ${ }^{35}$ The QD photoluminescence properties are well described by the set of rate equations including the bright and dark exciton, and the biexciton as represented in Fig. 3 .

The population transfer between the bright and the dark exciton states is governed by the two temperature-dependent rates $\gamma_{d}$ and $\gamma_{u}$ (see Fig. 3). We assume that these transitions are assisted by acoustic phonons whose energy matches the X-DX energy splitting $\Delta E$. At a temperature $T$ the number $N$ of acoustic phonons per quantum state of energy $\Delta E$ is given by the Bose-Einstein statistics and reads

$$
N=\frac{1}{\exp \left(\Delta E / k_{B} T\right)-1} .
$$

The downward transition rate $\gamma_{d}$ from the $\mathrm{X}$ to the DX state corresponds to the spontaneous and stimulated emission of a phonon, whereas the upward rate $\gamma_{u}$ corresponds to the absorption of a phonon. ${ }^{34}$ They are given by

$$
\begin{gathered}
\gamma_{d}=(N+1) \gamma_{0}, \\
\gamma_{u}=N \gamma_{0},
\end{gathered}
$$

where $\gamma_{0}$ is the zero-temperature downward rate $(N=0)$.

We have performed time-resolved photoluminescence of the $\mathrm{X}$ state at different temperatures. The results are presented in Fig. 7. The decay time of the $\mathrm{X}$ level depends not only on the radiative decay rate $\gamma_{\mathrm{X}}$ but also on the temperature-dependent $\gamma_{d}$ and $\gamma_{u}$ rates between the bright and dark excitons. At low temperature $(T=4 \mathrm{~K})$, the luminescence exhibits a fast monoexponential decay with a time scale of the order of $1 /\left(\gamma_{\mathrm{X}}+\gamma_{d}\right)$ corresponding to the radiative decay and the leakage toward the DX state. For intermediate temperatures $(T=20 \mathrm{~K}$ and $T=40 \mathrm{~K})$, the fast decay is still present and there is the apparition of a slow time scale 


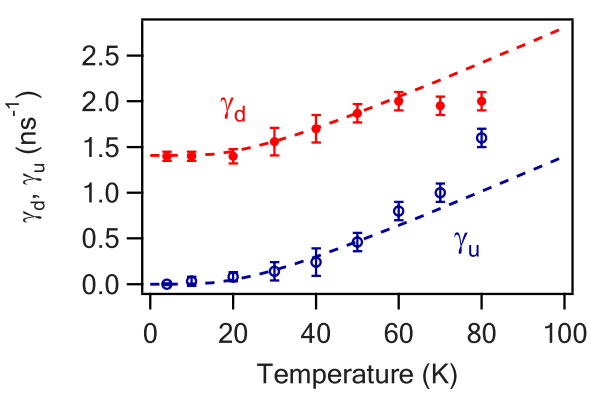

FIG. 8. (Color online) Downward and upward spin-flip rates between $\mathrm{X}$ and $\mathrm{DX}$ as a function of temperature. The dotted lines are the fits using Eqs. (1) and (2).

corresponding to the thermally activated reloading of the $\mathrm{X}$ state from the long lived DX state. For higher temperature $(T=80 \mathrm{~K})$, the reloading from the dark to the bright exciton becomes even more efficient and the decay appears as monoexponential with a time scale intermediate between the previous slow and fast time scales.

By fitting the lifetimes using the model of Fig. 3 we can extract the values for $\gamma_{\mathrm{X}}, \gamma_{\mathrm{NR}}, \gamma_{d}$, and $\gamma_{u}$. Each of these parameters has a specific influence on the shape of the luminescence decay and can be evaluated with a good precision. The radiative decay rate of the exciton is found to be $\gamma_{\mathrm{X}}$ $=1.4 \mathrm{~ns}^{-1}$ and is assumed to be temperature independent up to $T=60 \mathrm{~K}$ as it was observed for $\mathrm{CdSe} / \mathrm{ZnSe}$ self-assembled QDs. ${ }^{36}$ This assumption is justified a posteriori by the quality of the fits up to this temperature. The values for $\gamma_{d}$ and $\gamma_{u}$ are plotted in Fig. 8 as a function of temperature. This temperature dependence is well fitted using Eqs. (1) and (2). This enables us to obtain the DX-X energy splitting $\Delta E$ $=(6.0 \pm 0.2) \mathrm{meV}$ and the zero-temperature downward rate $\gamma_{0}=(1.41 \pm 0.02) \mathrm{ns}^{-1}$.

The rather large value for the DX-X energy splitting $\Delta E$ is an indication of the strong exciton confinement within the QD, owing to its relatively small size and the absence of a wetting layer. ${ }^{37}$ The bulk DX-X energy splitting for CdSe is $0.12 \mathrm{meV}$. According to the calculation performed by Klingshirn et al. ${ }^{38}$ confinement-induced enhancement of this splitting reaches a factor of 50 (that is $\Delta E=6 \mathrm{meV}$ for CdSe) for infinite barriers cylindrical dots of radius corresponding to the Bohr radius $a_{B}$ and of height corresponding to $a_{B} / 4$. These dimensions are compatible with the measured diameter $(10 \mathrm{~nm})$ of the NW and with the height expected from the CdSe growth duration. The DX-X energy splitting has been measured at $1.9 \mathrm{meV}$ for self-assembled $\mathrm{CdSe} / \mathrm{ZnSe}$ QD. ${ }^{37}$ The larger value that we have observed is an indication of the larger confinement in NWs owing to the absence of a wetting layer. This $\Delta E=6 \mathrm{meV}$ splitting corresponds to the value for very small (about $2 \mathrm{~nm}$ diameter) colloidal spherical CdSe nanocrystal as reported in Ref. 39. The value for zero-temperature downward transition rate $\gamma_{0}=1.4 \mathrm{~ns}^{-1}$ is comparable to what has been obtained for colloidal $\mathrm{CdSe}$ nanocrystals ${ }^{34}$ or some self-assembled InP/GaInP QDs. ${ }^{35}$ Slower rates $\left(\gamma_{0}=0.01 \mathrm{~ns}^{-1}\right)$ have also been observed in InGaAs/GaAs self-assembled QDs. ${ }^{40}$

A good fitting of the experimental data requires the inclusion of an effective nonradiative decay rate $\gamma_{\mathrm{NR}}$ of the DX state. This rate slightly increases with temperature from $\gamma_{\mathrm{NR}}=0.2 \mathrm{~ns}^{-1}$ at $4 \mathrm{~K}$ up to $\gamma_{\mathrm{NR}}=0.5 \mathrm{~ns}^{-1}$ at $80 \mathrm{~K}$. These values are of similar order of magnitude that what was reported in InGaAs/GaAs self-assembled QDs. ${ }^{40}$ Nonradiative phenomena in nanocrystals are generally slower ranging from hundreds of nanoseconds to a few microseconds in colloidal nanocrystals. ${ }^{34,41}$

\section{CONCLUSION}

In conclusion, we have used photon correlation spectroscopy to characterize a light-emitting QD embedded in a NW. We obtained a very good fit to the experimental data with a model based on a standard excitonic level scheme. This allowed us to extract quite complete dynamics of the neutral and charged excitons including charge hopping between these two states of the QD. We have performed temperaturedependent lifetime measurements of a CdSe QD embedded in a $\mathrm{ZnSe}$ NW. A careful quantitative analysis of these data has allowed us to confirm the strong influence of the dark exciton and to extract the dark-bright exciton splitting together with the transition rates between these two levels. The rather large dark-bright exciton splitting that we have measured is a signature of the strong confinement of the exciton within the QD in this NW geometry. This value is three times larger than for self-assembled CdSe QDs although the excitonic energy and the $\mathrm{X}-\mathrm{XX}$ and $\mathrm{X}-\mathrm{CX}$ energy splittings are of same order of magnitude.

CdSe/ZnSe QDs in NWs are nano-objects situated between self-assembled QDs and CdSe-based colloidal nanocrystals. ${ }^{42,43}$ The latter operate at room temperature but have a blinking problem and a lifetime above $20 \mathrm{~ns}$. On the other hand, self-assembled QDs are nonblinking and feature a subnanosecond lifetime allowing $\mathrm{GHz}$ repetition rates. QDs embedded in NWs have the potential to combine the best of both worlds by offering nonblinking room-temperature ${ }^{13}$ single photon sources with a high repetition rate. Furthermore, the versatility of this particular nanostructure growth technique offers interesting perspectives for engineering semiconducting QDs such as coupled QDs or waveguide coupled QDs.

\section{ACKNOWLEDGMENTS}

We thank M. Richard for many fruitful discussions, F. Donatini for very efficient technical support, and R. Cox for a careful reading of the paper. T.A. acknowledges support by Deutscher Akademischer Austauschdienst (DAAD). Part of this work was supported by European project QAP (Contract No. 15848). 
*Present address: Physics Institute, Humboldt University, Berlin, Germany; aichele@ physik.hu-berlin.de

†jean-philippe.poizat@grenoble.cnrs.fr

${ }^{1}$ X. Duan, Y. Huang, Y. Cui, J. Wang, and C. M. Lieber, Nature (London) 409, 66 (2001).

${ }^{2}$ W. Lu and C. M. Lieber, Nature Mater. 6, 841 (2007).

${ }^{3}$ C. Thelander, T. Martensson, M. T. Björk, B. J. Ohlsson, M. W. Larsson, L. R. Wallenberg, and L. Samuelson, Appl. Phys. Lett. 83, 2052 (2003).

${ }^{4}$ R. Könenkamp, Robert C. Word, and C. Schlegel, Appl. Phys. Lett. 85, 6004 (2004).

${ }^{5}$ H. M. Kim, Y. H. Cho, H. Lee, S. I. Kim, S. R. Ryu, D. Y. Kim, T. W. Kang, and K. S. Chung, Nano Lett. 4, 1059 (2004).

${ }^{6}$ X. Duan, Y. Huang, R. Agarwal, and C. M. Lieber, Nature (London) 421, 241 (2003).

${ }^{7}$ A. I. Hochbaum, R. Chen, R. D. Delgado, W. Liang, E. C. Garnett, M. Najarian, A. Majumdar, and P. Yang, Nature (London) 451, 163 (2008).

${ }^{8}$ Y. Cui, Q. Wei, H. Park, and C. M. Lieber, Science 293, 1289 (2001).

${ }^{9}$ M. S. Gudiksen, L. Lauhon, J. Wang, D. C. Smith, and C. M. Lieber, Nature (London) 415, 617 (2002).

${ }^{10}$ M. T. Björk, B. J. Ohlsson, T. Sass, A. I. Persson, C. Thelander, M. H. Magnusson, K. Deppert, L. R. Wallenberg, and L. Samuelson, Nano Lett. 2, 87 (2002); Appl. Phys. Lett. 80, 1058 (2002).

${ }^{11}$ C. Yang, Z. Zhong, and C. M. Lieber, Science 310, 1304 (2005).

${ }^{12}$ M. T. Borgström, V. Zwiller, E. Müller, and A. Imamoglu, Nano Lett. 5, 1439 (2005).

${ }^{13}$ A. Tribu, G. Sallen, T. Aichele, R. André, J.-Ph. Poizat, C. Bougerol, S. Tatarenko, and K. Kheng, Nano Lett. 8, 4326 (2008).

${ }^{14}$ P. Michler, A. Kiraz, C. Becher, W. V. Schoenfeld, P. M. Petroff, L. Zhang, E. Hu, and A. Imamoglu, Science 290, 2282 (2000).

${ }^{15}$ I. Fushman, D. Englund, A. Faraon, N. Stoltz, P. Petroff, and J. Vuckovic, Science 320, 769 (2008).

${ }^{16}$ R. Hanson and D. D. Awschalom, Nature (London) 453, 1043 (2008).

${ }^{17}$ P. J. Pauzauskie and P. Yang, Mater. Today 9, 36 (2006).

${ }^{18}$ N. Gregersen, T. R. Nielsen, J. Claudon, J. M. Gérard, and J. Mørk, Opt. Lett. 33, 1693 (2008).

${ }^{19}$ C. Simon, Y. M. Niquet, X. Caillet, J. Eymery, J. P. Poizat, and J. M. Gérard, Phys. Rev. B 75, 081302(R) (2007).

${ }^{20}$ A. Kiraz, S. Fälth, C. Becher, B. Gayral, W. V. Schoenfeld, P. M. Petroff, Lidong Zhang, E. Hu, and A. Imamoğlu, Phys. Rev. B 65, 161303(R) (2002).

${ }^{21}$ T. Aichele, A. Tribu, C. Bougerol, K. Kheng, R. André, and S. Tatarenko, Appl. Phys. Lett. 93, 143106 (2008).

${ }^{22}$ V. Türck, S. Rodt, R. Heitz, O. Stier, M. Strassburg, U. W. Pohl, and D. Bimberg, Phys. Status Solidi B 224, 217 (2001).

${ }^{23}$ B. Patton, W. Langbein, and U. Woggon, Phys. Rev. B 68, 125316 (2003).

${ }^{24}$ G. Bacher, R. Weigand, J. Seufert, V. D. Kulakovskii, N. A. Gippius, A. Forchel, K. Leonardi, and D. Hommel, Phys. Rev. Lett. 83, 4417 (1999); F. Gindele, K. Hild, W. Langbein, and U. Woggon, Phys. Rev. B 60, R2157 (1999); F. Gindele, U. Wog- gon, W. Langbein, J. M. Hvam, K. Leonardi, D. Hommel, and H. Selke, ibid. 60, 8773 (1999); J. C. Kim, H. Rho, L. M. Smith, Howard E. Jackson, S. Lee, M. Dobrowolska, and J. K. Furdyna, Appl. Phys. Lett. 75, 214 (1999); T. Kümmell, R. Weigand, G. Bacher, A. Forchel, K. Leonardi, D. Hommel, and H. Selke, ibid. 73, 3105 (1998); S. M. Ulrich, S. Strauf, P. Michler, G. Bacher and A. Forchel, ibid. 83, 1848 (2003); V. D. Kulakovskii, G. Bacher, R. Weigand, T. Kümmell, A. Forchel, E. Borovitskaya, K. Leonardi, and D. Hommel, Phys. Rev. Lett. 82, 1780 (1999).

${ }^{25}$ G. Sallen, Ph.D. thesis, Université Joseph Fourier, Grenoble, 2009 (http://tel.archives-ouvertes.fr/tel-00362497/fr/).

${ }^{26}$ M. Bayer and A. Forchel, Phys. Rev. B 65, 041308(R) (2002).

${ }^{27}$ J. Wang, M. S. Gudiksen, X. Duan, Y. Cui, and C. M. Lieber, Science 293, 1455 (2001).

${ }^{28}$ Y. M. Niquet and D. C. Mojica, Phys. Rev. B 77, 115316 (2008).

${ }^{29}$ M. H. M. van Weert, N. Akopian, F. Kelkensberg, U. Perinetti, M. P. van Kouwen, J. Gómez Rivas, M. T. Borgström, R. E. Algra, M. A. Verheijen, E. P. A. M. Bakkers, L. P. Kouwenhoven, and V. Zwiller, arXiv:0808.2908, Small (to be published).

${ }^{30}$ C. Santori, D. Fattal, J. Vučković, G. S. Solomon, E. Waks, and Y. Yamamoto, Phys. Rev. B 69, 205324 (2004).

${ }^{31}$ M. H. Baier, A. Malko, E. Pelucchi, D. Y. Oberli, and E. Kapon, Phys. Rev. B 73, 205321 (2006).

${ }^{32}$ J. Suffczyński, T. Kazimierczuk, M. Goryca, B. Piechal, A. Trajnerowicz, K. Kowalik, P. Kossacki, A. Golnik, K. P. Korona, M. Nawrocki, J. A. Gaj, and G. Karczewski, Phys. Rev. B 74, 085319 (2006).

${ }^{33}$ E. Moreau, I. Robert, L. Manin, V. Thierry-Mieg, J. M. Gérard, and I. Abram, Phys. Rev. Lett. 87, 183601 (2001).

${ }^{34}$ O. Labeau, P. Tamarat, and B. Lounis, Phys. Rev. Lett. 90, 257404 (2003).

${ }^{35}$ M. Reischle, G. J. Beirne, R. Roßbach, M. Jetter, and P. Michler, Phys. Rev. Lett. 101, 146402 (2008).

${ }^{36}$ I. C. Robin, R. André, Le Si Dang, H. Mariette, S. Tatarenko, J. M. Gérard, K. Kheng, F. Tinjod, M. Bartels, K. Lischka, and D. Schikora, Phys. Status Solidi B 241, 542 (2004); I. C. Robin, R. André, and J. M. Gérard, Phys. Rev. B 74, 155318 (2006).

${ }^{37}$ J. Puls, M. Rabe, H.-J. Wünsche, and F. Henneberger, Phys. Rev. B 60, R16303 (1999).

${ }^{38}$ C. Klingshirn, M. Hetterich, J. M. Hvam, W. Langbein, U. Woggon, and F. Gindele, Solid State Commun. 106, 653 (1998).

${ }^{39}$ Al. L. Efros, M. Rosen, M. Kuno, M. Nirmal, D. J. Norris, and M. Bawendi, Phys. Rev. B 54, 4843 (1996).

${ }^{40}$ J. M. Smith, P. A. Dalgarno, R. J. Warburton, A. O. Govorov, K. Karrai, B. D. Gerardot, and P. M. Petroff, Phys. Rev. Lett. 94, 197402 (2005).

${ }^{41}$ M. Nirmal, D. J. Norris, M. Kuno, M. G. Bawendi, A. L. Efros, and M. Rosen, Phys. Rev. Lett. 75, 3728 (1995).

${ }^{42}$ P. Michler, A. Imamoğlu, M. D. Mason, P. J. Carson, G. F. Strouse, and S. K. Buratto, Nature (London) 406, 968 (2000).

${ }^{43}$ B. Mahler, P. Spinicelli, S. Buil, X. Quelin, J. P. Hermier, and B. Dubertet, Nature Mater. 7, 659 (2008). 